\title{
Diagnostic value of the Hs-cTnT/CysC ratio in patients with stage $\geq 3$ CKD and acute myocardial infarction
}

\section{Lihua Yang}

First Hospital of Medical College of Shantou University https://orcid.org/0000-0003-2469-0117

\section{Cudan Cai}

First Hospital of Medical College of Shantou University

Hanfei Lin

First Hospital of Medical College of Shantou University

Jianwei Peng

First Hospital of Medical College of Shantou University

\section{Yanqiang Peng}

First Hospital of Medical College of Shantou University

Kefei Wu ( $\nabla$ wukefei2012@163.com )

https://orcid.org/0000-0002-9403-9656

\section{Research article}

Keywords: Acute myocardial infarction, Chronic kidney disease, hs-cTnT, Renal insufficiency, Troponin

Posted Date: August 27th, 2020

DOl: https://doi.org/10.21203/rs.3.rs-59606/v1

License: (9) (i) This work is licensed under a Creative Commons Attribution 4.0 International License. Read Full License 


\section{The study's first author: Lihua Yang}

Lihua Yang Department of Nephrology, The First Affiliated Hospital of Shantou University Medical College, NO. 57, Changping Road, Shantou City, Guangdong Prov. China. E-mail:ylh9524@126.com Mobile: 0086-13425313741

Chudan Cai Department of Nephrology, The First Affiliated Hospital of Shantou University Medical College, NO. 57, Changping Road, Shantou City, Guangdong Prov. China. E-mail:ccd1512@163.com Mobile: 0086-13076333326

Hanfei Lin Department of Nephrology, The First Affiliated Hospital of Shantou University Medical College, NO. 57, Changping Road, Shantou City, Guangdong Prov. China. E-mail:hanfeilin@hotmail.com Mobile:0086-13502717903

Jianwei Peng Department of Nephrology, The First Affiliated Hospital of Shantou University Medical College, NO. 57, Changping Road, Shantou City, Guangdong Prov. China. E-mail:598650502@qq.com Mobile: 0086-13794126245

Yanqiang Peng, Department of Nephrology, The First Affiliated Hospital of Shantou University Medical College, NO. 57, Changping Road, Shantou City, Guangdong Province, China. Tel: 86-0754-88905621, E-mail: pengyqgzsums@163.com

\section{Corresponding author:}

Kefei Wu Department of Nephrology, The First Affiliated Hospital of Shantou University Medical College, NO. 57, Changping Road, Shantou City, Guangdong Prov. China. E-mail: wukefei2012@163.com Mobile: 0086-13536820427 Zip code: 515041 


\title{
Diagnostic value of the Hs-cTnT/CysC ratio in patients with stage $\geq 3$ CKD and acute myocardial infarction
}

\author{
Abstract \\ Background: We investigated the diagnostic value of the ratio of high sensitivity \\ troponin to cystatin $\mathrm{C}$ in $\mathrm{CKD}$ patients with stage $\geq 3$ chronic kidney disease (CKD) \\ and acute myocardial infarction.
}

Methods: We retrospectively analyzed 401 patients with suspected acute myocardial infarction (AMI) who underwent coronary angiography in the chest pain center at our hospital during 2013-2019. Among the 196 patients studied, 113 were placed in an AMI group and 83 in a non-AMI group.

Results: There were no significant differences in age, sex, or the presence of hypertension, diabetes, gout/hyperuricemia, stroke, tumor, or epidermal growth factor receptors between the two groups $(\mathrm{P}>0.05)$. A correlation analysis showed that there was a positive correlation between $\mathrm{Cys} C$ and $\mathrm{Cr}$, with a correlation coefficient of $0.872(\mathrm{P}<0.001)$. A receiver operating characteristic curve for the high-sensitivity cardiac troponin $\mathrm{T}$ (hs-c TnT)/CysC ratio showed an area under the curve value of $0.925(\mathrm{P}<0.001)$, with sensitivity of $78.4 \%$ and specificity of $94.0 \%$.

Conclusion: The hs-TnT/CysC ratio can thus be used as an index to predict AMI in patients with stage $\geq 3 \mathrm{CKD}$.

Keywords: Acute myocardial infarction, Chronic kidney disease, hs-cTnT, Renal insufficiency, Troponin 


\section{Background}

The incidence of acute myocardial infarction (AMI) is higher in patients with chronic kidney disease $(\mathrm{CKD})$ than in those without $\mathrm{it}^{1,2}$. Rapid diagnosis and treatment are important for a positive prognosis in AMI patients. AMI diagnosis depends on the clinical manifestations, myocardial markers, electrocardiography (ECG) results, and other indicators, among which troponin is the most convenient, specific, and sensitive. Troponin, which can be detected by a new generation of highly sensitive methods, is helpful for diagnosing minimal myocardial injury and contributing to an early diagnosis of $\mathrm{AMI}^{3}$.

At present, the most widely used highly sensitive techniques can detect highly-sensitive clinical troponin T (hs-cTnT) and highly-sensitive troponin I (hs-TnI). These high-sensitivity detection abilities have improved sensitivity and thus can detect increases in troponin in many non-ischemic myocardial diseases as well, including renal failure ${ }^{4,5}$, stroke ${ }^{6}$, pulmonary embolism ${ }^{7}$, and critical diseases ${ }^{8}$. Clinically, increased hs-cTnT is observed in many asymptomatic renal insufficiency patients with normal ECGs ${ }^{9,10}$, which may be related to minimal myocardial injury and a decreased estimated glomerular filtration rate (eGFR) $)^{11}$.Study found that cTnT forms in ESRD patients are small $(<18 \mathrm{kDa})$ and different from forms seen in AMI patients ${ }^{12}$, however, the current test can not distinguish them. Because this test is also affected by renal function, however, it cannot be an effective marker to measure the degree of myocardial injury. Although dynamic observation of ECG changes and troponin levels could be highly important for the diagnosis, it would undoubtedly 
increase the patient's economic and medical burden. To date, there is no consensus on how to define the troponin level of patients with renal insufficiency when myocardial injury occurs ${ }^{13}$. Some studies have suggested that the formula for conversion of troponin should be used, but the formula is too complicated to be widely used in clinical practice. Therefore, this study aimed to find more effective indicators for diagnosing AMI.

\section{methods}

\section{Data source}

We retrospectively analyzed 401 patients with suspected AMI who had undergone coronary angiography after admission to the chest pain center at our hospital between January 2013 and August 2019. In all, 196 patients (151 men, 45 women) were included in the study. The clinical data and laboratory indicators of the patients were collected, including the age and sex of the patients and whether they had a history of hypertension, abnormal blood lipid levels, diabetes, stroke, gout/hyperuricemia, malignant tumor, or other serious disease. The laboratory indexes included hs-cTnT, creatine kinase-membrane-bound (CK-MB) isoenzyme, myoglobin, hemoglobin, albumin, Cr, CysC, uric acid cholesterol, triglycerides, low-density lipoprotein (LDL), and high-density lipoprotein

\section{Research methods}

Inclusion criteria: $\geq$ stage $3 \mathrm{CKD}$ according to the Modification of Diet in Renal 
Disease formula: eGFR $<60 \mathrm{~mL} / \mathrm{min} / 1.73 \mathrm{~m}^{2}$ for $\geq 3$ months with or without evidence of kidney injury.

AMI diagnostic criteria: (1) type 1 myocardial infarction (fourth universal myocardial infarction definition, established in 2018) ${ }^{14}$; (2) cTn increased and/or decreased; (3) $>99 \%$ upper reference limit (URL )at least once; (4) accompanied by at least one of the following: AMI symptoms, new ECG changes indicating ischemia, development of a pathological Q wave, imaging evidence of new loss of viable myocardium or new regional wall motion abnormality in a pattern consistent with an ischemic etiology, identification of a coronary thrombus by angiography including intracoronary imaging or at autopsy.

Exclusion criteria: definitively diagnosed myocarditis, acute kidney injury, hemolysis, advanced malignant tumor, disseminated intravascular coagulation.

\section{Methods}

The subjects were divided into an AMI group $(n=113)$ and a non-AMI group $(n=83)$. Age, sex, creatinine, CKD stage, hypertension, blood lipid levels, diabetes, stroke, gout/hyperuricemia, tumor history, hemoglobin, albumin, Cr, CysC, UA, cholesterol, triglycerides, LDL, HDL, and cholesterol were compared between the two groups. The basic data and the diagnostic values of $\mathrm{cTnT}$, cTnT/CysC ratio, CK-MB isoenzyme, and myoglobin for AMI were compared. Cobas/e170 hs-cTnT (Roche, 
Indianapolis, IN, USA) was used to detect hs-cTnT. The blood collection time was $<10$ min for both outpatients with chest pain and hospitalized patients with chest pain. Routine blood and biochemical tests were performed using Beckman Coulter (Brea, CA, USA) instruments.

\section{Statistical methods}

SPSS 19.0 software (IBM, Armonk, NY, USA) was used for statistical analysis. The continuous variables in accordance with normal distribution were expressed as means \pm standard deviations. The differences between the two groups were analyzed using an independent sample $t$ test. Non-normal variables were expressed by medians (interquartile intervals), and the differences between the groups were analyzed using rank sum tests (Mann-Whitney rank sum test for between the two groups).

Categorical variables were expressed as percentages). The differences between groups were tested using the $\mathrm{R} \times \mathrm{C} \chi^{2}$ test. Pearson's and Spearman's correlation coefficients were calculated to analyze the correlation of variables. A binary logistic regression analysis was used to correct the study indexes, and the $95 \%$ confidence interval was calculated. Receiver operating characteristic (ROC) curves and areas under the curves (AUCs) were used to evaluate the diagnostic value of the technique. A value of $\mathrm{P}<$ 0.05 indicated statistical significance.

\section{Results}

\section{Basic characteristics of clinical data}

There were no significant differences in age, sex, hypertension, diabetes, 
gout/hyperuricemia, stroke, tumor, or eGFR between the AMI and non-AMI groups $(\mathrm{P}>0.05 ;$ Table 1).

\section{Results of laboratory indexes}

The differences in the cholesterol, LDL, and CK-MB isoenzyme values in the AMI and non-AMI groups were statistically significant $(\mathrm{P}<0.05)$. Conversely, the hemoglobin, albumin, $\mathrm{Cr}$, and $\mathrm{CysC}$ values were not significantly different between the groups $(\mathrm{P}>0.05$; Table 2$)$.

\section{Correlation analysis of $\mathrm{CysC}$ and $\mathrm{Cr}$, age, and sex}

CysC was positively correlated with $\mathrm{Cr}$, with a correlation coefficient of $0.872(\mathrm{P}<$ 0.001; Table 3, Fig.1).

\section{ROC curve and interactive dot graph}

The reference value of hs-cTnT is $14 \mathrm{ng} / \mathrm{L}$ according to the 99 th percentile value provided by the manufacturer. We created an interactive dot graph based on this value. When hs-cTnT was $14 \mathrm{ng} / \mathrm{L}$, the diagnostic sensitivity was $100 \%$, whereas the specificity was only $12 \%$ (Fig.2). The ROC curve was created based on the hs-cTnT/CysC ratio (Fig.3). The AUC value was $0.925(\mathrm{P}<0.001)$; optimal cutoff point was 234.0 ; sensitivity was $78.4 \%$; specificity was $94.0 \%$; and the Yoden index was 0.724 . The ROC curves were compared according to the hs-cTnT, hs-cTnT/CysC ratio, CK-MB isoenzymes, and myoglobin values. Comparisons of the hs-TNT value with the hs-cTnT/CysC ratio, CK-MB isoenzymes, and myoglobin were statistically significant $(\mathrm{P}=0.046, \mathrm{P}<0.001$, and $\mathrm{P}<0.001$, respectively $)$, as were the comparison between the hs-cTnT/CysC ratio with $\mathrm{CK}-\mathrm{MB}$ isoenzymes and myoglobin $(\mathrm{P}=0.002$ 
and $\mathrm{P}=0.003$, respectively; Fig.4, Table 4). There was no significant difference between the CK-MB isoenzymes and myoglobin $(\mathrm{P}>0.05)$.

\section{Discussion}

Troponin is a marker that can be detected in the blood when myocardial injury occurs.

When AMI has developed, enough troponin is released into the bloodstream that a significant increase can be detected in the peripheral blood. With the improved detection method, hs-cTNT can now be identified within 1-3 h after myocardial injury. A disadvantage is that hs-cTNT can be increased for other reasons in patients without myocardial injury. Among them, patients with renal insufficiency are the most common $^{4}$, in whom the hs-sTNT is negatively correlated with the eGFR. At present, the reason for the increased troponin in patients with renal insufficiency is not clear. Some studies proposed that it may be related to myocardial injury and declining renal excretion function.

It is generally believed, therefore, that a diagnosis cannot rely only on a single increase of troponin (99th percentile)—dynamic observation would be more significant $^{15,16}$. Continuous monitoring of hs-cTnT (during 3 to $6 \mathrm{~h}$ intervals) is helpful for distinguishing acute myocardial ischemia from noncardiac diseases ${ }^{17}$. If the change in hs-cTnT is $<20 \%$, chronic injury is more likely. There have been many asymptomatic patients with renal insufficiency, however, whose ECG changes are not enough to diagnose AMI. According to the guidelines and specifications, these patients should be under dynamic observation of changes in both their ECG and troponin. This protocol, though, increases the economic and medical burdens of the 
patients and even delays treatment of patients who might have developed AMI. The optimal hs-cTnT cutoff value for patients with renal insufficiency is still uncertain. Many studies have proposed various correction methods, but they are too complex for clinical application and thus cannot be popularized.

The incidence of cardiovascular disease in CKD patients is high ${ }^{18}$, which may be related to some common pathogenesis shared by CHD and CKD. AMI is an dangerous cardiovascular disease that requires rapid diagnosis. Coronary angiography is the gold standard for diagnosing AMI and the key to treatment. Patients with renal insufficiency, however, are more likely to develop contrast-induced nephropathy than normal people ${ }^{19}$, and may need emergent dialysis. To avoid CKD progression, we should pay attention to the possibility of AMI and avoid unnecessary coronary angiography.

$\mathrm{CysC}$ is a low-molecular-weight protein composed of 122 amino acids that is cleared only by the kidney. The CysC concentration depends on the GFR in the kidney. It is unrelated to the patient's age, sex, height, age, inflammatory reaction, or tumor presence ${ }^{20}$, among other factors. Therefore, increased CysC indicates impaired renal function and is therefore a measure that is more sensitive and accurate than $\mathrm{Cr}$ for predicting renal function. Some studies have found that $\mathrm{Cys} C$ may be an independent predictor of metabolic syndrome and is related to coronary atherosclerosis ${ }^{21,22}$. The possible mechanism for such a relation is that $\mathrm{Cys} C$ participates in activity regulation of cysteine protease and matrix metalloproteinase. It also participates in the pathological processes of atherosclerosis, AMI, and other cardiovascular diseases. 
Studies found that concentration of CysC was decreased ${ }^{23}$ or slightly higher ${ }^{24,25}$ in AMI patients than in healthy controls group, but in CKD patients it was significantly increased. Therefore, increased $\mathrm{Cys} C$ and troponin $\mathrm{T}$ can be detected in patients with CKD due to the decreased eGFR.

This study found that the hs-cTnT/CysC ratio is more specific than any other method for diagnosing AMI in patients with CKD of $\geq$ stage 3 . The rate is significantly higher than that of hs-cTnT for diagnosing AMI. Apparently, the hs-cTnT/CysC ratio can offset some of the impact of the decreased eGFR.

Because this study was performed in a single-center, was retrospective, and had a small sample size, the proportion of stage 5 CKD patients was low. Hence, our results should be further verified by a study with a larger patient sample. We should also determine how the protocol works in apparently healthy people.

\section{Conclusion}

The Hs-cTnT/CysC ratio can be used to predict AMI in patients with $\geq$ stage $3 \mathrm{CKD}$. This finding is of great significance as it allows early diagnosis of AMI. 


\section{List of abbreviations}

$\begin{array}{ll}\text { AMI } & \text { acute myocardial infarction } \\ \text { CK-MB } & \text { creatine kinase membrane-bound } \\ \text { Cr } & \text { creatinine } \\ \text { CysC } & \text { cystatin C } \\ \text { ECG } & \text { electrocardiography } \\ \text { eGFR } & \text { estimated glomerular filtration rate } \\ \text { HDL } & \text { high-density lipoprotein } \\ \text { hs-cTnT } & \text { highly sensitive clinical troponin T } \\ \text { hs-TnI } & \text { highly sensitive troponin I } \\ \text { LDL } & \text { low-density lipoprotein } \\ \text { ROC } & \text { receiver operating characteristic } \\ \text { UA } & \text { uric acid }\end{array}$




\section{Declarations}

\section{Ethics approval and consent to participate}

The study was approved by the Ethics Committee of the First Affiliated Hospital of Shantou University Medical College.

\section{Consent for publication}

Not applicable.

\section{Consent for paticipate}

Not applicable.

\section{Availability of data and materials}

Not applicable.

\section{Code availability}

Not applicable.

\section{Competing interests}

All authors declare no relevant competing interests.

\section{Funding}

This work was supported by Science and Technology Planning Project of Guangdong Province(2014A020212289) to Dr Peng. The funders had no role in study design, data collection and analysis, decision to publish, or preparation of the manuscript.

\section{Authors' contributions}

WKF designed the research; YLH and CCD assisted in the research design; YLH conducted the research,PJW and LHF performed the statistical analysis;PYQ provided advice and verification for statistical analysis; YLH wrote the manuscript; YLH had the primary responsibility for the final content. All authors contributed to discussions of the analyses, critically reviewed the manuscript, and approved the final manuscript. 
All authors declare no relevant conflicts of interest.

\section{Authors' information (optional)}

Affiliations

Department of Nephrology the first The First Affiliated Hospital of Shantou

University Medical College, shantou, 515000, China

Lihua Yang* Cudan Cai Hanfei Lin Jianwei Peng Yanqiang Peng Kefei $\mathrm{Wu}^{\#}$

Department of Nephrology, The First Affiliated Hospital of Shantou University Medical College, Shantou, China.

Lihua Yang Department of Nephrology, The First Affiliated Hospital of Shantou University Medical College, NO. 57, Changping Road, Shantou City, Guangdong Prov. China. E-mail:ylh9524@126.comＭobile: 0086-13425313741

Chudan Cai Department of Nephrology, The First Affiliated Hospital of Shantou University Medical College, NO. 57, Changping Road, Shantou City, Guangdong Prov. China. E-mail:ccd1512@163.com Mobile: 0086-13076333326

Hanfei Lin Department of Nephrology, The First Affiliated Hospital of Shantou University Medical College, NO. 57, Changping Road, Shantou City, Guangdong Prov. China. E-mail:hanfeilin@hotmail.com Mobile: 0086-13502717903

Jianwei Peng Department of Nephrology, The First Affiliated Hospital of Shantou University Medical College, NO. 57, Changping Road, Shantou City, Guangdong Prov. China. E-mail:598650502@qq.com Mobile: 0086-13794126245 Yanqiang Peng, Department of Nephrology, The First Affiliated Hospital of Shantou University Medical College, NO. 57, Changping Road, Shantou City, Guangdong 
Province, China. Tel: 86-0754-88905621, E-mail: pengyqgzsums@163.com

Corresponding author

Kefei Wu, Department of Nephrology, The First Affiliated Hospital of Shantou

University Medical College, NO. 57, Changping Road, Shantou City, Guangdong

Province, China. Tel: 86-0754-88905621, E-mail: wukefei2012@163.com

\section{Acknowledgment}

We thank Nancy Schatken BS, MT(ASCP), from Liwen Bianji, Edanz Group China (www.liwenbianji.cn/ac), for editing the English text of a draft of this manuscript. 


\section{References}

1. Lascasas, J. et al. Demographic, clinical characteristics and cardiovascular disease burden in a Portuguese cohort of older chronic kidney disease patients. J Bras Nefrol 41, 29-37 (2019).

2. Ku, E. \& Mitsnefes, M. M. Cardiovascular disease in young adults with incident ESRD. Nat Rev Nephrol 15, 390-391 (2019).

3. Chapman, A. R. et al. High-Sensitivity Cardiac Troponin and the Universal Definition of Myocardial Infarction. Circulation 141, 161-171 (2020).

4. Musso, P., Cox, I., Vidano, E., Zambon, D. \& Panteghini, M. Cardiac troponin elevations in chronic renal failure: Prevalence and clinical significance. Clin Biochem 32, 125-130 (1999).

5. Croitoru, M. \& Taegtmeyer, H. Spurious rises in troponin T in end-stage renal disease. Lancet 346, 974 (1995).

6. Král, M. et al. Troponin T in acute ischemic stroke. Am J Cardiol 112, 117-121 (2013).

7. Daquarti, G., March Vecchio, N., Mitrione, C. S., Furmento, J., Ametrano, M. C., Dominguez Pace, M. P. \& Costabel, J. P. High-sensitivity troponin and right ventricular function in acute pulmonary embolism. Am J Emerg Med 34, 1579-1582 (2016).

8. Stein, R., Gupta, B., Agarwal, S., Golub, J., Bhutani, D., Rosman, A. \& Eng, C. Prognostic implications of normal $(<0.10 \mathrm{ng} / \mathrm{ml})$ and borderline $(0.10$ to $1.49 \mathrm{ng} / \mathrm{ml})$ troponin elevation levels in critically ill patients without acute coronary syndrome. Am J Cardiol 102, 509-512 (2008).

9. Needham, D. M., Shufelt, K. A., Tomlinson, G., Scholey, J. W. \& Newton, G. E. Troponin I and T levels in renal failure patients without acute coronary syndrome: a systematic review of the literature. Can J Cardiol 20, 1212-1218 (2004).

10. Irfan, A. et al. Determinants of high-sensitivity troponin T among patients with a noncardiac cause of chest pain. Am J Med 125, 491-498.e1 (2012).

11. Huang, H. et al. Diagnosis of acute myocardial infarction in patients with renal insufficiency using high-sensitivity troponin T. Clin Chem Lab Med 53, 723-730 (2015).

12. Mingels, A. M. A. et al. Cardiac troponin T: Smaller molecules in patients with end-stage renal disease than after onset of acute myocardial infarction. Clin Chem 63, 683-690 (2017).

13. deFilippi, C. \& Seliger, S. The Cardiac Troponin Renal Disease Diagnostic Conundrum: Past, Present, and Future. Circulation 137, 452-454 (2018).

14. Thygesen, K. et al. Fourth Universal Definition of Myocardial Infarction (2018). Circulation 138 e618-618e651 (2018).

15. Twerenbold, R. et al. 0/1-Hour Triage Algorithm for Myocardial Infarction in Patients With Renal Dysfunction. Circulation 137, 436-451 (2018).

16. Kraus, D. et al. Cardiac Troponins for the Diagnosis of Acute Myocardial Infarction in Chronic Kidney Disease. J Am Heart Assoc 7, e008032 (2018).

17. Roffi, M. et al. 2015 ESC Guidelines for the management of acute coronary syndromes in patients presenting without persistent ST-segment elevation: Task Force for the Management of Acute Coronary Syndromes in Patients Presenting without Persistent ST-Segment Elevation of the European Society of Cardiology (ESC). Eur Heart J 37, 267-315 (2016).

18. Navarro, M. A., Gosch, K. L., Spertus, J. A., Rumsfeld, J. S. \& Ho, P. M. Chronic Kidney Disease and Health Status Outcomes Following Acute Myocardial Infarction. J Am Heart Assoc 5, (2016). 
19. Silvain, J. et al. Contrast-induced acute kidney injury and mortality in ST elevation myocardial infarction treated with primary percutaneous coronary intervention. Heart 104, 767-772 (2018).

20. Levey, A. S. \& Inker, L. A. Assessment of Glomerular Filtration Rate in Health and Disease: A State of the Art Review. Clin Pharmacol Ther 102, 405-419 (2017).

21. Mao, Q. et al. Association of Cystatin C with Metabolic Syndrome and Its Prognostic Performance in Non-ST-Segment Elevation Acute Coronary Syndrome with Preserved Renal Function. Biomed Res Int 2019, 8541402 (2019).

22. Hoke, M. et al. Cystatin C and the risk for cardiovascular events in patients with asymptomatic carotid atherosclerosis. Stroke 41, 674-679 (2010).

23. Noto, D. et al. Cystatin C levels are decreased in acute myocardial infarction: effect of cystatin C G73A gene polymorphism on plasma levels. Int J Cardiol 101, 213-217 (2005).

24. Abid, L., Charfeddine, S., Kammoun, S., Turki, M. \& Ayedi, F. Cystatin C: A prognostic marker after myocardial infarction in patients without chronic kidney disease. J Saudi Heart Assoc 28, 144-151 (2016).

25. Negrusz-Kawecka, M., Poręba, R., Hulok, A., Sciborski, K., Marczak, J. \& Bańkowski, T. Evaluation of the significance of cystatin C levels in patients suffering from coronary artery disease. Adv Clin Exp Med 23, 551-558 (2014). 
Figures

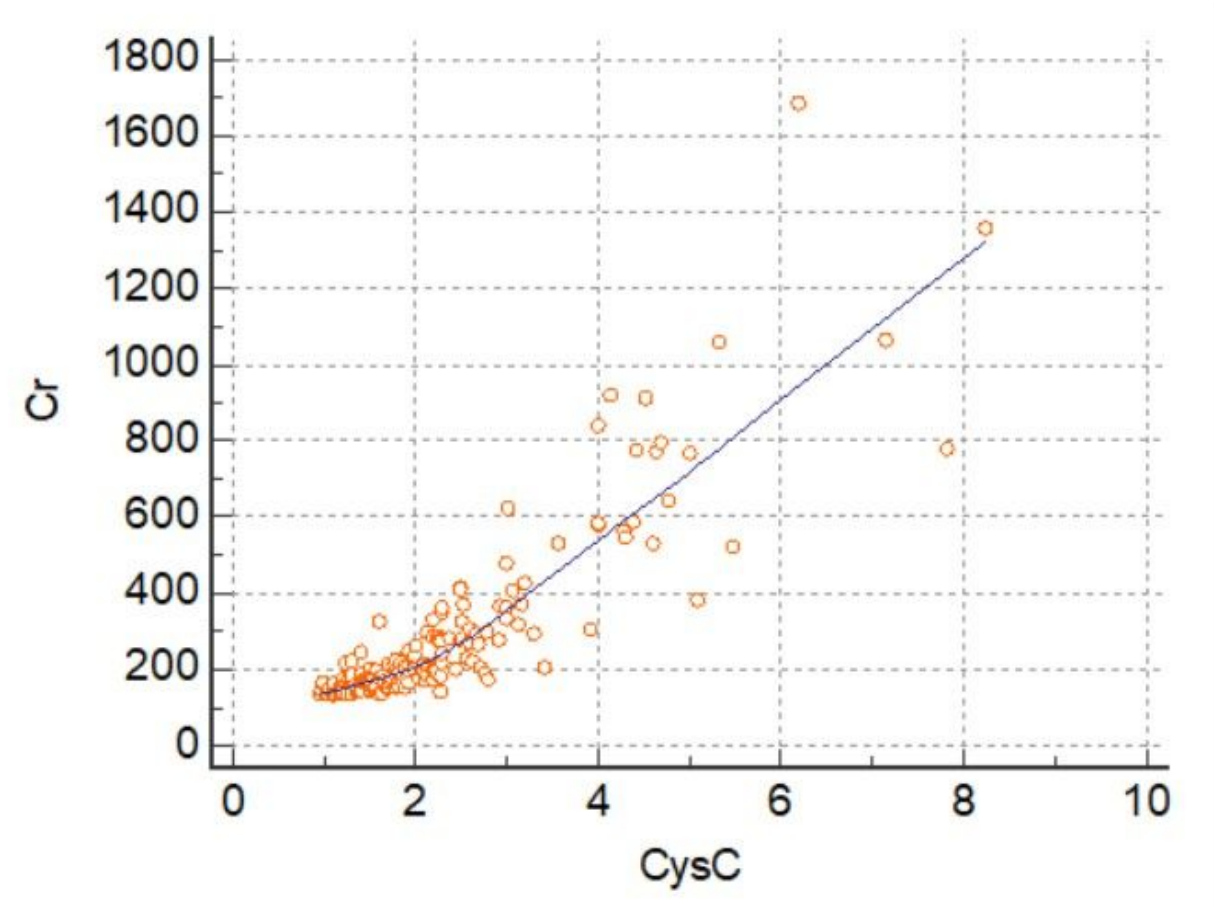

Figure 1

CysC was positively correlated with $\mathrm{Cr}$, with a correlation coefficient of $0.872(\mathrm{P}<0.001$; Table 3, Fig.1). 


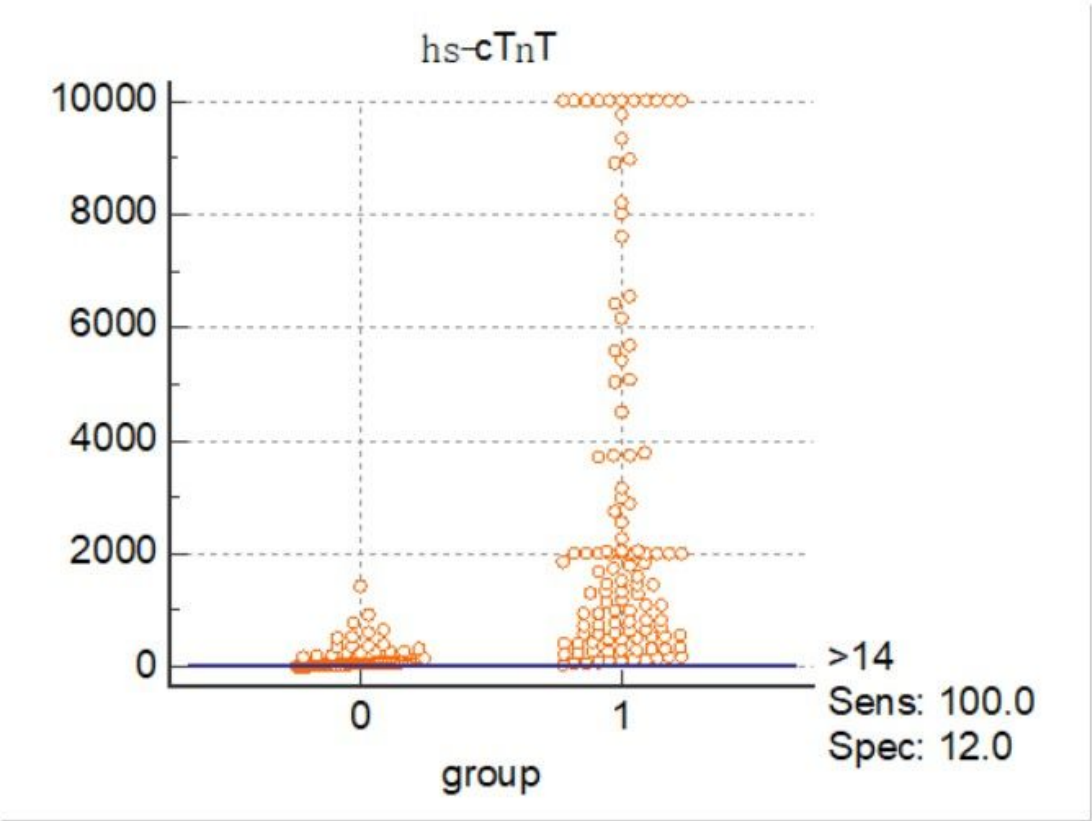

Figure 2

The reference value of hs-cTnT is $14 \mathrm{ng} / \mathrm{L}$ according to the 99th percentile value provided by the manufacturer. We created an interactive dot graph based on this value. When hs-cTnT was $14 \mathrm{ng} / \mathrm{L}$, the diagnostic sensitivity was $100 \%$, whereas the specificity was only $12 \%$ (Fig.2). 


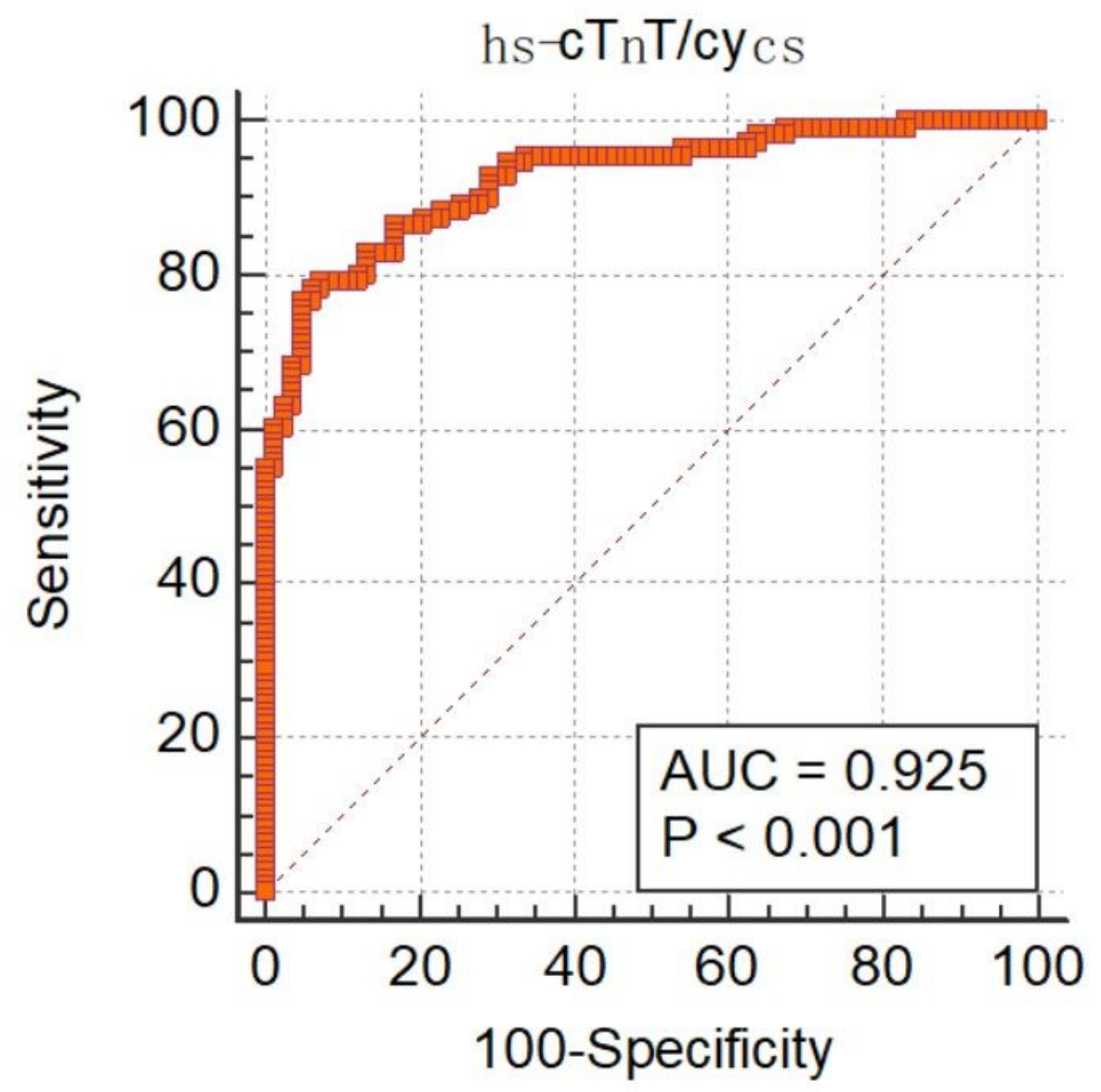

Figure 3

The ROC curve was created based on the hs-cTnT/CysC ratio (Fig.3). 


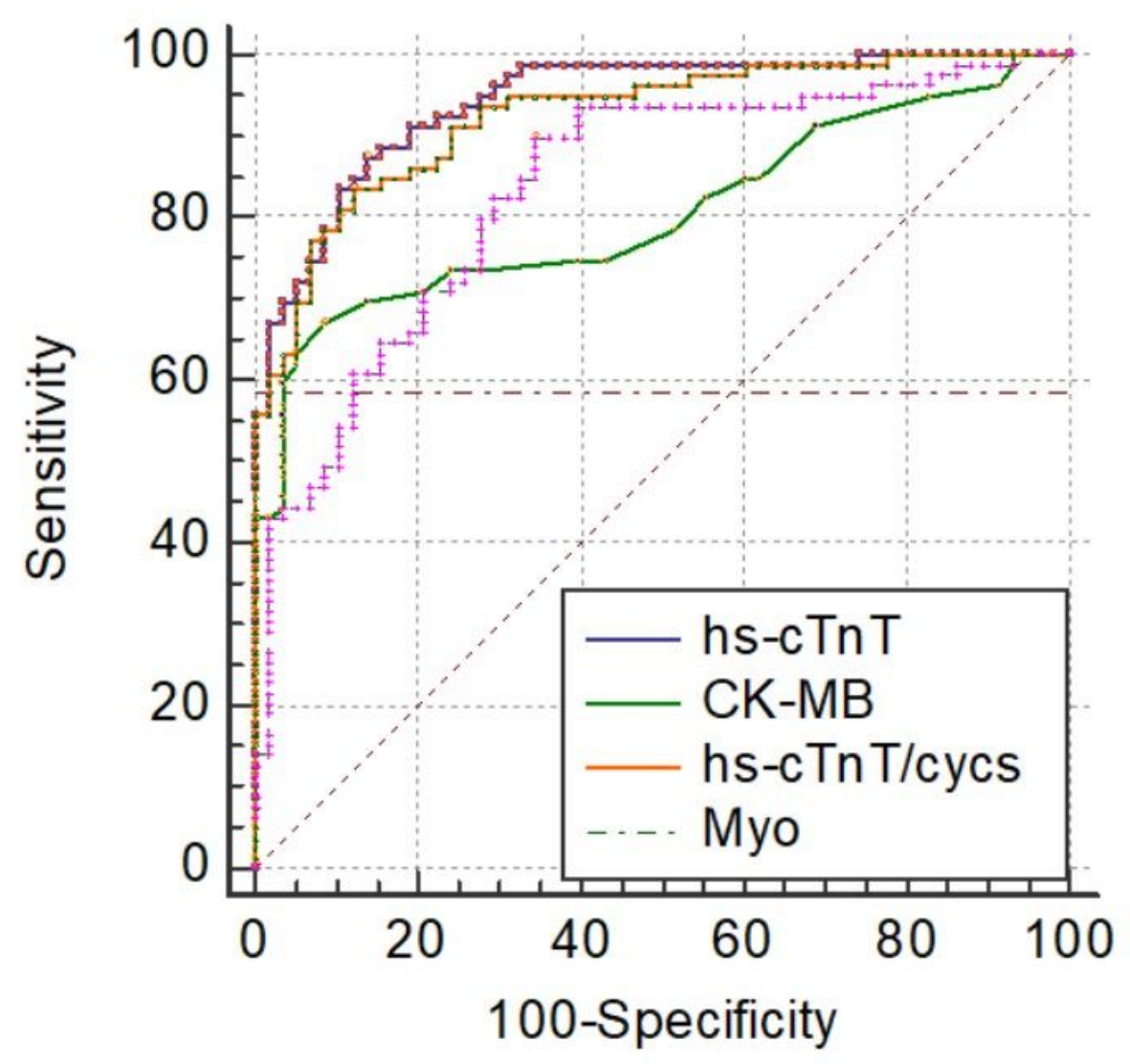

Figure 4

The ROC curves were compared according to the hs-cTnT, hs-cTnT/CysC ratio, CK-MB isoenzymes, and myoglobin values. Comparisons of the hs-TNT value with the hs-cTnT/CysC ratio, CK-MB isoenzymes, and myoglobin were statistically significant $(P=0.046, P<0.001$, and $P<0.001$, respectively), as were the comparison between the hs-cTnT/CysC ratio with CK-MB isoenzymes and myoglobin $(\mathrm{P}=0.002$ and $\mathrm{P}=$ 0.003 , respectively; Fig.4, Table 4). There was no significant difference between the CK-MB isoenzymes and myoglobin $(P>0.05)$.

\section{Supplementary Files}

This is a list of supplementary files associated with this preprint. Click to download.

- Table1.jpg

- Table3.jpg

- Table4.jpg 
- Table2.jpg 